\title{
EXTENDED DETECTORS IN NEUTRON TIME-OF-FLIGHT DIFFRACTION EXPERIMENTS
}

\author{
J. M. CARPENTER
}

Department of Nuclear Engineering, The University of Michigan, Ann Arbor, Michigun, USA

Received II July 1966

In the method of neutron time-of-flight diffraction, ${ }^{1,2}$ ) which has been applied at several laboratories $^{3-8}$ ) a chopped polyenergetic neutron beam is scattered from a target to a detector erected at scattering angle $\theta_{n}$. Elastically-scattered neutrons of speed $t$ arrive at a detector at time $t$ given by

$$
t=\left[L^{\prime}+L\left(\theta_{\mathrm{s}}\right)\right] / v
$$

where $L^{\prime}$ is the distance from the chopper to the sample, and $L\left(\theta_{\mathrm{s}}\right)$ is the distance from the sample to the detector at scattering angle $\theta_{\mathrm{s}}$. The Bragg condition giving the wavenumber $k$ of neutrons scattered from reciprocal lattice vector $\tau$ may be written

$$
\begin{array}{r}
2 k \sin \left(\frac{1}{2} \theta_{\mathrm{s}}\right)=2 \pi|\tau|, \\
k=2 \pi|\tau| /\left[2 \sin \left(\frac{1}{2} \theta_{\mathrm{s}}\right)\right] .
\end{array}
$$

Since $r=\hbar k / m$, the time of arrival is

$$
t=2(m / h)(2 \pi|\tau|)^{-1}\left[L^{\prime}+L\left(\theta_{\mathrm{s}}\right)\right] \sin \left(\frac{1}{2} \theta_{\mathrm{s}}\right) .
$$

From eq. (3) it is clear that $t$ may be made independent of $\theta_{\text {s }}$ for fixed $|\boldsymbol{\tau}|$ if :

$$
\begin{aligned}
{\left[L^{\prime}+L\left(\theta_{\mathrm{s}}\right)\right] \sin \left(\frac{1}{2} \theta_{\mathrm{s}}\right) } & =\text { constant, } \\
& =L^{\prime}+L(\pi)
\end{aligned}
$$

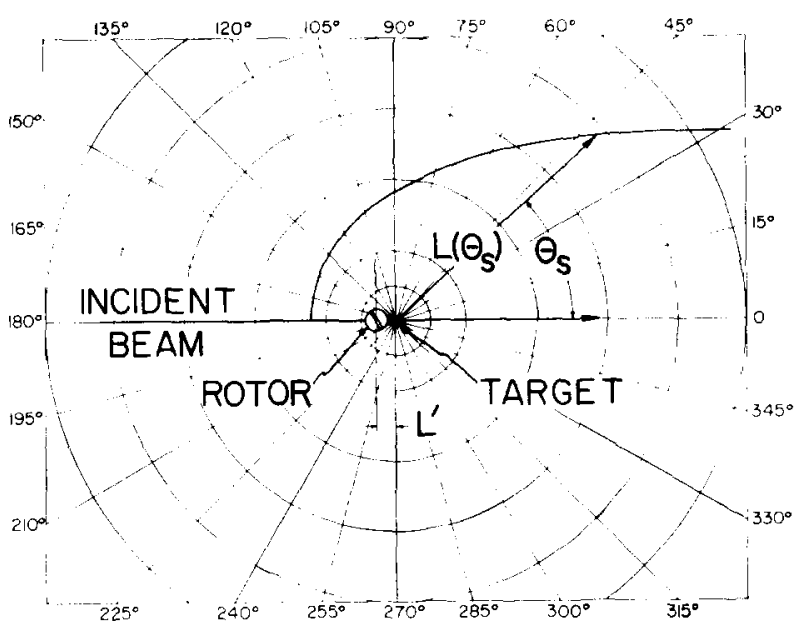

Fig. 1. The locus of constant time of arrival for neutron timeof-flight diffraction, for $L^{\prime}-0.3 \mathrm{~m}, L(\pi)=1.2 \mathrm{~m}$.

and then independently of $\theta_{\mathrm{s}}$.

$$
t=2(m / h)\left[L^{\prime}+L(\pi)\right](2 \pi|\tau|)^{-1} .
$$

The locus of constant time of arrival is then

$$
L\left(0_{\mathrm{s}}\right)=\left[L^{\prime}+L(\pi)\right] / \sin \left(\frac{1}{2} 0_{\mathrm{s}}\right)-L^{\prime} .
$$

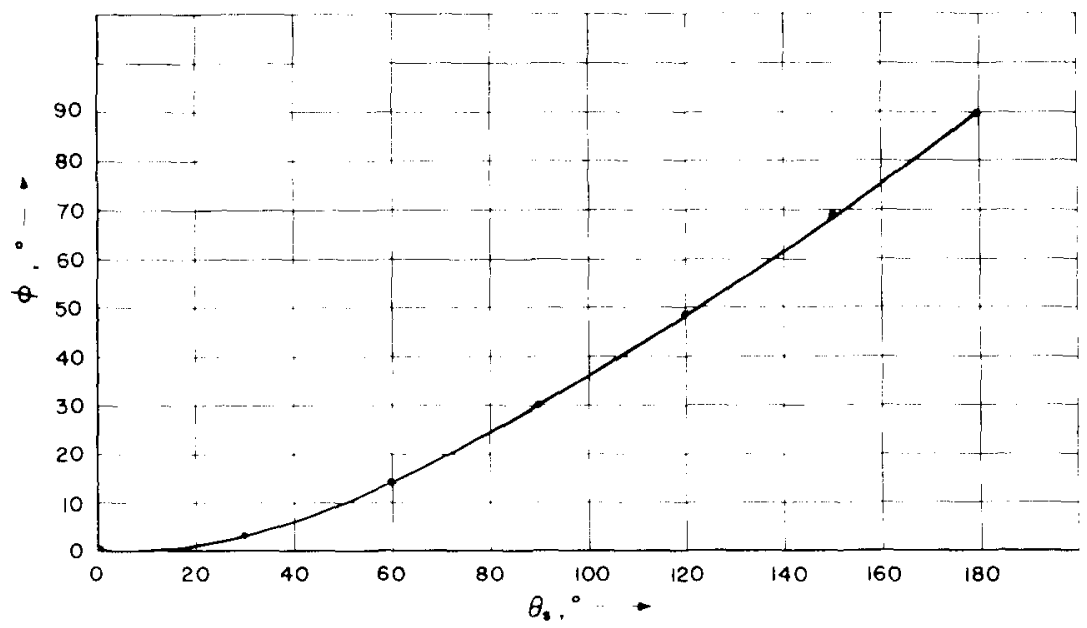

Fig. 2. The tangent angle between the locus of constant time of arrival, and the incident beam direction, for $L^{\prime}=0.3 \mathrm{~m}$, $L(\pi)=1.2 \mathrm{~m}$ 
The angle $\phi$ between the tangent to the locus (6) and the incident beam direction is

$\phi=\theta_{\mathrm{s}}-\tan ^{-1}\left\{2 L\left(\theta_{\mathrm{s}}\right) \tan \left(\frac{1}{2} \theta_{\mathrm{s}}\right) /\left[L^{\prime}+L\left(\theta_{\mathrm{s}}\right)\right]\right\}$.

The locus of detectors for constant time of arrival given by eq. (6) is shown in fig. 1, for flight path lengths $L^{\prime}=0.3 \mathrm{~m}$ and $L(\pi)=1.2 \mathrm{~m}$. The tangent angle $\phi$ is shown in fig. 2 for the same choice.

Two inferences may be drawn. First, if a large array of detectors is placed on the locus (6), then the solid angle subtended by the detectors at the target may be increased with consequent increase in counting rate. Identical neutron wavelengths do not carry the information to each $0_{\mathrm{s}}$, but all neutrons scattered by planes of a given spacing $\tau^{-1}$ will arrive simultaneously. Second, for best attainable resolution, any detector array should be situated tangent to the locus (6).

Using an extended bank of detectors would somewhat complicate extraction of structure factors, but this disadvantage would be compensated by the higher counting rates. A material with known structure factors may be used to obtain an intensity calibration.

Brugger ${ }^{9}$ ) has erected two small banks of detectors at points on the locus of eq. (6). Buras ${ }^{5}$ ) uses a circular are of detectors for single crystal work. but notes that some reflections may overlap on the time scale. This difficulty would be resolved by the present method.

\section{References}

1) G. R. Ringo, in Handhud der Phwih 32 (Springer, 1957) p. 590 .

-) P. A. Egelstaft, Proc. Symp. Neutron Time-of-Flight Methods. Saclay, 1961 (IAEA. Vienna, 1961) 71.

3) B. Buras and J. Leciejewicz, Nuklconika 81196.3175.

4) B. Buras et al., Nukleonika 9 (1964) 523.

j) B. Buras et al., Risö Report $106(1965)$.

(i) L. H. Schwartz, Bull. Am. Phys. Soc. 11, 10 (1965) 1122.

7) R. M. Brugger, IDO 17170 (1966).

๙) J. D. Sutton, private communication (1966).

9 ) R. M. Brugger, private communication (1966). 